\title{
Data Replication for the Distributed Database Using Decision Support Systems
}

\author{
P.Elango \\ Research Scholar \\ Allagappa University \\ Karaikudi, India
}

\author{
Dr.K.Kuppusamy \\ Professor and Head \\ Allagappa University \\ Karaikudi, India
}

\author{
N.Prabhu \\ Assistant Professor \\ Gobi Arts and Science \\ College \\ Gobichettipalayam, India
}

\author{
Dr.N.M.Mallika \\ Dean and Assistant \\ Professor \\ Sri Vasavi college \\ Erode, India
}

\begin{abstract}
Replication is a subject of enthusiasm for the conveyed figuring, disseminated frameworks, and database networks. Choice emotionally supportive networks ended up pragmatic with the advancement of minicomputer, timeshare working frameworks and appropriated figuring. Reproduced information may get lacking because of framework disappointment, adaptation to internal failure, and unwavering quality. A halfway replication is quantized in the replication framework will expand the non repeated framework. Adaptation to internal failure is the property that empowers a framework (regularly PC based) to keep working legitimately. Exchange Processing Replication (TP-R) and Decision-bolster replication diagram (DDS-R) will clear the non copy and it is utilized to clear the server issues and framework blunder. This procedure is top notch in disseminated frameworks and it doesn't neglect to distinguish the framework blunders when different accesses are multiplexed.
\end{abstract}

Keywords: Cloud computing, Data mining, Data replication, Partial replication, cascades replication.

\section{INTRODUCTION}

Replication is the way toward sharing data in order to guarantee consistency between repetitive assets, for example, programming or equipment parts, to improve dependability, adaptation to non-critical failure, or availability. It could be information replication if similar information is put away on various capacity gadgets or calculation replication if a similar figuring task is executed commonly. It is the procedure of naturally conveying duplicates of information and database objects among SQL Server examples, and keeping the dispersed data synchronized. Replication is the way toward sharing data in order to guarantee consistency between excess assets, for example, programming or equipment segments, to improve dependability, adaptation to internal failure, or openness. It could be information replication if similar information is put away on numerous capacity gadgets or calculation replication if a similar processing task is executed ordinarily. The safe sharing of data in this kind of condition is an unpredictable issue. The proprietors of the distinctive information sources will have diverse strategies on access to and the scattering of the information that they hold. There are two principle sorts of replication conventions: dynamic replication, in which all imitations forms simultaneously all info messages. Aloof replication, in which just a solitary one of the reproductions forms all information messages and intermittently transmits its present state to different copies so as to keep up consistency from the previous years, Distributed Databases have taken consideration in the database look into network. Information dispersion and replication offer open doors for improving execution through parallel inquiry execution and burden adjusting just as expanding the accessibility of information. In an appropriated database framework, information are frequently recreated to improve unwavering quality and accessibility, subsequently expanding its trustworthiness.

\section{DATA REPLICATION}

Replication is the way toward sharing data to guarantee consistency between excess assets, for example, programming or equipment segments to improve dependability, adaptation to internal failure, or availability. It could be information replication if similar information is put away on numerous capacity gadgets or calculation replication if a similar registering task is executed ordinarily. Replication has been contemplated in numerous regions, particularly in appropriated frameworks (essentially for adaptation to internal failure purposes) and in databases (principally for execution reasons). Replication is one of the most seasoned and most critical subjects in the general territory of disseminated framework. A critical issue in circulated frameworks is the replication of information. Information are for the most part recreated to upgrade dependability or improve execution. Replication is the way toward duplicating information from an information store or document framework to various PCs to synchronize the information. Database replication is ending up progressively imperative job for database applications. Duplicated information are ending up increasingly more of intrigue recently. Replication is a financially savvy approach to build accessibility and utilized for both execution and blame tolerant purposes in this way presenting a steady tradeoff among consistency and productivity. Replication gives reinforcement database extensive endeavors as a rule have locales where it is basic to get to information consistently. In the event that a server breakdown it is critical to approach similar information on an alternate server and this typically requires authoritative mediation. Database replication is rapidly turning into a basic device for giving high accessibility, survivability, and elite for database applications. Be that as it may, to give helpful replication one needs to take care of the non-inconsequential issue of keeping up information consistency between every one of the reproductions. Replication utilizes the accompanying three servers to be specific Publisher, Distributor, and Subscriber. The essential issue with information replication is that an update to some random intelligent item should be spread to all put away duplicates of that object. A trouble that emerges quickly is that a few locales holding a duplicate of the item may be inaccessible (on account of a site or system disappointment) at the season of the update. The conspicuous procedure of spreading refreshes quickly to all duplicates is in 
this manner likely inadmissible, in light of the fact that it infers that the update and along these lines the exchange will fall flat if any of those duplicates is at present inaccessible. It might be said, truth be told, information is less accessible under this technique than it would be in the non-imitated case.

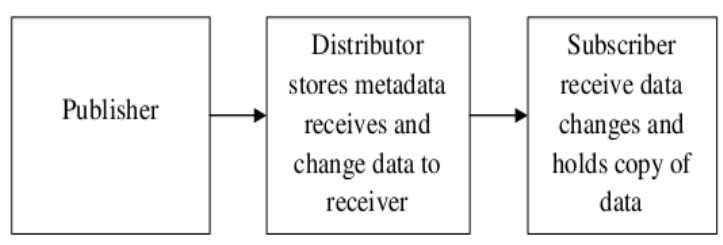

Figure. 1 Replication Process

\section{ANALYTICAL MODEL FOR PARTIAL REPLICATION}

Give us a chance to accept that a non recreated database has a preparing limit, that a non-reproduced database can execute exchanges per time unit. All destinations have a similar limit, a non-recreated database in which the whole handling limit is utilized for executing neighborhood exchanges, however locales in the repeated database need to utilize a portion of its preparing limit with respect to coordination with different destinations. We term the coordination fill in as remote work. Along these lines, each site in a recreated database utilizes a small amount of its handling limit with regards to nearby work and the rest of the limit with respect to remote work, for example at that point, the nearby work performed in a site is

$$
L i=c-R i
$$

The scale out is the sum of the amount of local work executed at each site, divided by the processing capacity of a nonreplicated database $\frac{S \text { caleout }=\sum{ }^{n} i=1^{L i}}{C}$ i.e. how many times the capacity of a non-replicated system is increased when it is replicated. The more local work each site executes, the better the scalability of the system. The total amount of local work at site is the sum of accesses to objects stored at (Eq. 2). The objects stored at site are defined by the function

$$
r(i, k) \quad L_{i}=\sum_{K=1}^{0} C \cdot r(i, k) \cdot a_{i} k, \forall=1 \ldots n
$$

\section{TRANSACTION PROCESSING REPLICATION (TP-R)}

Value-based replication is a sort of replication that enables information adjustments to be proliferated steadily between servers in a dispersed situation. Value-based replication can be utilized for a wide range of uses, from revealing servers and information warehousing situations to Web servers and web based business applications. Value-based replication is utilized at a significant number of the prevalent Web locales on the Internet that run SQL Server, including MSN.com, Passport.com, Barnes and Noble.com, and Buy.com. Valuebased replication is an adaptable and solid answer for dispersing information in elite situations. An exchange handling replication (TP-R) approach that can keep up close ongoing exchange respectability at information duplicate locales is fundamental. In languid replication, exchanges are executed first at one imitation. Any updates are spread to different reproductions simply after exchange submits, therefore giving quick reaction times. The idea of exchange was first presented in database frameworks, with the goal of supporting the steady execution of simultaneous tasks over shared information However, from that point forward exchanges have been connected substantially more extensively, e.g., in dispersed frameworks in numerous application situations, in which they improve unwavering quality and certification information consistency. This is about the exchange submit for recreated databases. There is work here for certain years, with creators proposing the usage of deliberations, regularly used to indicate dependable conveyed frameworks (e.g., agreement, all out request multicast) to help crash blame tolerant database replication or, all the more conventionally, exchange handling. TP-R replication is essentially worried about making a solitary picture of a database crosswise over appropriated selfgoverning destinations and saving database honesty in close continuous preparing. The general uprightness of databases is safeguarded by sending information changes coming about because of single client exchanges.

\section{CASCADING REPLICATES}

Course conduct is a Pareto improvement over conduct in which person's base choices just on their private data, since a course mirrors a coordination of more private data than any single individual has.

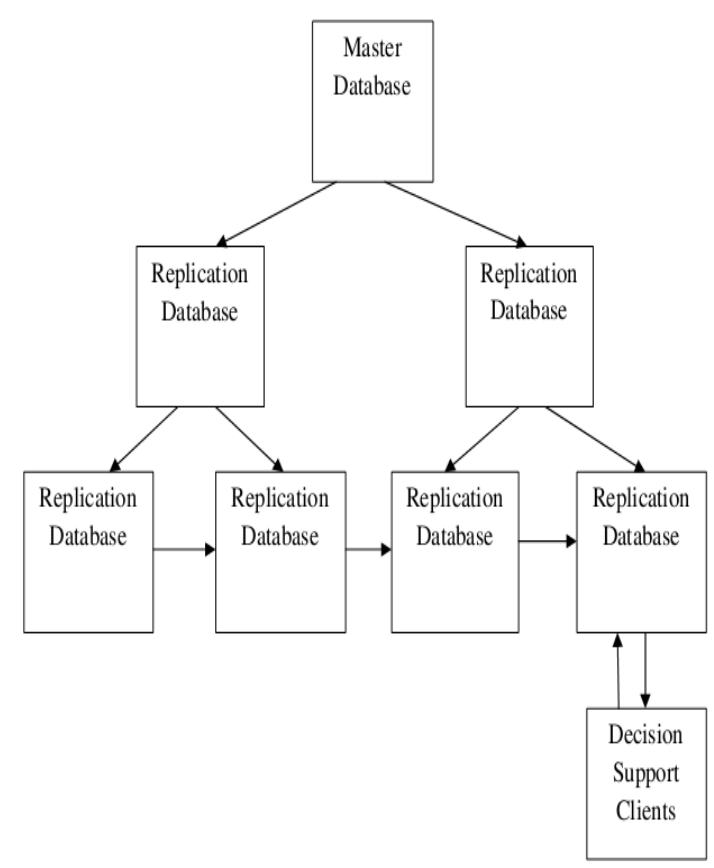




\section{DECISION-SUPPORT REPLICATION SCHEMA (DDS-R)}

DSS-R ways to deal with replication generally are based on different innovation varieties of table duplicating. The common choice help application has a prerequisite for reliable period information sources and not really for information that is regularly updated current. DSS-R approaches, at that point, don't commonly stress over keeping the information current. Predictable, stable information for a given period is the most astounding necessity for these sorts of utilizations. The choice emotionally supportive networks are tuned for inquiry preparing, commonly by including more lists. For this situation, at that point, persistent proliferation of updates would meddle with the capacity of the inquiry apparatus to give sensible execution. The replication server ought to give different planning choices which can make duplicates dependent on coordinated occasions (clock or interim), on application occasions (for example end of day compromise finished), or on manual solicitation. Other vital prerequisites for choice help incorporate the capacity to get to inheritance generation framework information from sources, for example, IMS, RMS, VSAM, and level documents and to give complex information control/improvement to that information.

\section{DSS-R SCHEMA}

The esteem added to the information by control or improvement is imperative in DSS-R conditions. Sources are commonly inheritance frameworks and the replication arrangement ought to give the capacity to rebuild the information from heritage groups into the social model. Apparatuses ought to offer help for joining information from various sources, for computing new qualities, for accumulating information and for changing encoded information into spellbinding structures. A vital side point to remember is that one of the key advantages of DSS-R, accumulation of information or de standardization, is something that ought not to be done when the duplicate is updatable.

\section{RESULT AND DISCUSSION}

In our proposed strategy, the information replication for choice supporting framework is portrayed. The usage was done in JAVA. Here in our proposed part, the manufactured database is utilized. Timeouts are best when a huge level of the transient disappointments can be overlooked, which is reliant on the downtime appropriation. Be that as it may, for strength to stay high, the normal hub lifetime should be fundamentally more prominent than the timeout. To assess this situation where timeouts ought to have sway, we played out a trial utilizing an engineered follow where we fluctuated the fix edge and the hub timeout. Since the framework would perceive hubs returning after a perpetual disappointment and promptly terminate all pending timeouts for these hubs, we allotted new characters to such hubs to permit long timeouts to lapse ordinarily. Table1 demonstrates the consequences of this reenactment the all out bytes sent as a component of timeout

\begin{tabular}{|r|r|r|}
\hline $\begin{array}{l}\text { Time Out } \\
\text { (hours) }\end{array}$ & Full Replication & Partial Replication \\
\hline $\mathbf{0 . 1}$ & 18 & 25 \\
\hline $\mathbf{1}$ & 15 & 22 \\
\hline $\mathbf{1 0}$ & 12 & 19 \\
\hline $\mathbf{1 0 0}$ & 10 & 16 \\
\hline $\mathbf{1 0 0 0}$ & 8 & 12 \\
\hline $\mathbf{1 0 0 0 0}$ & 4 & 10 \\
\hline
\end{tabular}

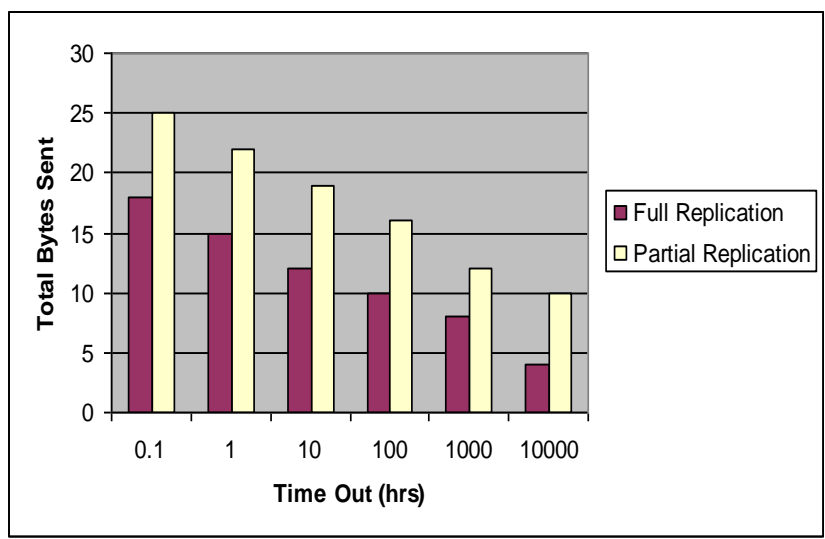

Figure 3 Comparison of time out on bandwidth

The impact of timeouts on bandwidth is compared with full and partial replication using decision support system. Figure 3 shows the number of copies created for various timeout values.

Table2 Comparison of Full and Partial Replication based on Fault occurrence rate and System error

\begin{tabular}{|c|c|c|}
\hline Methods & $\begin{array}{c}\text { Fault } \\
\text { Occurrence } \\
\text { Rate }\end{array}$ & System error \\
\hline Full Replication & $75 \%$ & $25 \%$ \\
\hline Partial Replication & $45 \%$ & $10 \%$ \\
\hline
\end{tabular}




\section{ACKNOWLEDGMENTS}

Replication assumes a fundamental job in disseminated frameworks. Frameworks disappointments regularly happen in repeated information's because of the undesirable versatility and adaptation to internal failure. Choice Support frameworks (DSS) is utilized to clear the blame happens in the database by utilizing a halfway replication with explanatory model. It is to comprehend the potential adaptability increases of incomplete replication concerning full replication. It will give better execution by utilizing a DDS-R outline. It will destroy the framework mistakes and the circulated frameworks with database can access with various replication with no adaptation to non-critical failure. Exploratory Results unmistakably demonstrates the information replication for the conveyed database utilizing choice emotionally supportive networks will give the better execution by lessening the bogus blunders.

\section{REFERENCES}

[1] Reza Akbarinia, Mounir Tlili, Esther Pacitti, Patrick Valduriez and Alexandre A. B. lima," Replication in DHTs using Dynamic Groups," Lecture Notes in Computer Science, Vol. 6790, No.3, pp. 1-19, 2011.

[2] Reza Akbarinia, Mounir Tlili, Esther Pacitti, Patrick Valduriez and Alexandre A. B. lima," Continuous Time stamping for Efficient Replication Management in DHTs," Lecture Notes in Computer Science, Vol. 6265, pp. 38-49, 2010 .

[3] Prasanna Padmanabhan, Le Gruenwald, Anita Vallur and Mohammed Atiquzzaman," A survey of data replication techniques for mobile ad hoc network databases, International journal on very large database," Vol. 17, pp. 1143-1164, 2008.

[4] Dutta, H., Kamil, A., Pooleery, M., Sethumadhavan, S., Demme, J.: Distributed Storage of Large-Scale Multidimensional Electroencephalogram Data Using Hadoop and HBase," Grid and Cloud Database Management, pp. 331347, 2011.

[5] Hannes Muhleisen, Tilman Walther and Robert Tolksdorf," Data Location Optimization for a Self-Organized Distributed Storage System," In Proc. of the Third World Congress on Nature and Biologically Inspired Computing (NaBIC), pp. 176 - 182, Oct 2011.

[6] Yi Lin, Bettina Kemme, Marta Patino Martinez, Ricardo Jimenez Peris," Consistent Data Replication: Is it feasible in WANs," Information Science and Computer communication, 2004.

[7] Zahia Guessoum, Jean-Pierre Briot, Nora Faci and Olivier Marin," Towards reliable multi-agent systems: An adaptive replication mechanism," Journal of Multi agent and Grid Systems, Vol.6, No. 1, pp. 1-24, 2010.
[8] Byung-Gon Chun, Frank Dabek, Andreas Haeberlen, Emil Sit, Hakim Weatherspoon, M. Frans Kaashoek, John Kubiatowicz and Robert Morris," Efficient Replica Maintenance for Distributed Storage Systems," In Proc. of the Symposium on Networked Systems Design and Implementation, 2006.

[9] Michael H. Zack," The role of decision support systems in an indeterminate world," Decision Support Systems, Vol. 43, No. 4, pp. 1664-1674, 2007.

[10] Sanjay Kumar Tiwari and A.K.Sharma," Management Issues in Replicated Distributed Real Time Database," International Journal of Advance in Science and Technology, Vol.3, No1, pp. 75-89, 2011.

[11] Maris G. Martinsons and Robert M. Davison," Strategic decision making and support systems: Comparing American, Japanese and Chinese management," Decision Support Systems, Vol. 43, pp. $284-300,2007$.

[12] G. Bent, D. Vyvyan, David Wood, Petros Zerfos and Seraphin Calo," Distributed Policy Based Access to Networked Heterogeneous ISR Data Sources," In Proc. of the conference on International society of optics and photonics, Vol. 7694, 2010.

[13] H. T. Barney and G. C. Low," Object Allocation with Replication in Distributed Systems," World Academy of Science, Engineering and Technology, Vol. 48, pp. 558-566, 2008.

[14] Costel Ciuchi, Dan Picu and Gyorgy Todoran," Managing Knowledge and Data for a Better Decision in Public Administration," Administration and Public Management, Vol. 2011, No. 17, pp. 64-81, 2011.

[15] Carlo Curino, Evan Jones, Yang Zhang and Sam Madden," Schism: a Workload-Driven Approach to Database Replication and Partitioning," In Proc of the Conference on very large database Endowment, Vol.3, No.1, Sep 2010. 